\title{
Mechanical characterization and micromechanical modeling of bread dough.
}

\begin{abstract}
The mechanical behavior of dough, gluten, and starch was studied in an effort to investigate whether bread dough can be treated as a two phase (starch and gluten) composite material. Mechanical loading tests revealed rate-dependent behavior for both the starch and the gluten constituents of dough. There is evidence from cryo-scanning electron microscopy that damage in the form of debonding between starch and gluten occurs when the sample is stretched. In addition, the Lodge material model was found to deviate from the tension and shear stress-strain test data by a considerably larger amount than from the compression test data. This could indicate that "damage" is dominant along the gluten-starch interface, causing debonding; the latter occurs less under compression loading, but is more prevalent in tension and shear loading. A single-particle finite element model was developed using starch as a filler contained in a gluten matrix. The interface between starch and gluten was modeled using cohesive zone elements with damage/debonding occurring under opening/tension and sliding/shear modes. The numerical results are compared to experimental stress-strain data obtained at various loading conditions. A comparison of stress-strain curves obtained from 2D and 3D single-particle models and a 2D multiparticle model led to good agreement, indicating that the single-particle model can be used to adequately represent the microstructure of the dough studied here.
\end{abstract}

Keyword: composite materials, compressive strength, compressive testing, finite element analysis, micromechanics, scanning electron microscopy, shear strength, stressstrain relations, Bread dough 